\title{
Performance Bounds in OFDM Channel Prediction
}

\author{
Ian C. Wong and Brian L. Evans \\ Wireless Networking and Communications Group \\ Dept. of Electrical and Computer Engineering \\ 1 University Station C0803 \\ The University of Texas at Austin, Austin, Texas 78712 \\ Email: \{iwong,bevans\}@ece.utexas.edu
}

\begin{abstract}
OFDM channel prediction algorithms have been proposed to combat feedback delay in adaptive OFDM systems. However, no simple closed-form expression that relates prediction mean-square error (MSE) with the design parameters in OFDM channel prediction has been reported in the literature. This paper attempts to fill that gap by deriving simple closedform asymptotic lower bounds of the MSE for OFDM channel prediction in deterministic doubly-selective fading channels. The bounds relate the best-achievable MSE with the predictor design parameters in a simple manner, thus providing important insight into the design of OFDM channel predictors. We compare the bounds to the deterministic Cramer-Rao bound for parameters consistent with IEEE 802.16e, and show that the asymptotic bounds are reasonably tight for practical scenarios.
\end{abstract}

\section{INTRODUCTION}

Adaptive OFDM systems overcome the limitation of conventional OFDM by allowing the transmitter to vary the power, modulation, and code rate on each subcarrier depending on the current channel state information (CSI) [1]. This requires the transmitter to have knowledge of the CSI, which can be obtained through feedback from the receiver's channel estimates, or through its own estimates in a time division duplex (TDD) reciprocal channel. In high mobility environments, in which the Doppler frequency is high and the channel changes rapidly, the CSI used by the transmitter would be outdated due to the processing and feedback delays, thereby causing significant performance degradation [2].

One possible solution to the feedback delay problem is the concept of channel prediction, whereby previous estimates of the time-varying channel is used to predict the fading value at a future time instant. In recent years, OFDM channel prediction algorithms [3][4][5][6] have been proposed to combat the feedback delay problem for closed-loop link adaptation in OFDM systems. In the design of these channel prediction algorithms, the ability to compute upper bounds on the performance (or lower bounds on the error) of channel prediction as a function of these parameters is a very useful tool for the systems engineer to make appropriate design decisions. Furthermore, these bounds can also be used as a benchmark for all the different algorithms that have been proposed in the literature.

In [7], approximate mean-square error (MSE) bounds have been derived using a first-order Taylor approximation of the channel prediction MSE expression for a parametric time- and frequency-selective channel model. The Cramer-Rao bound
$(\mathrm{CRB})$ is then used to derive a lower bound on this MSE, and a simple approximate expression when the prediction length is large was derived. The effect of the number of timedomain pilot blocks on the MSE was investigated using these expressions. However, the effect of the pilot subcarriers in the frequency domain, and the pilot spacings in both time and frequency dimensions, is not apparent in their expressions.

In this paper, we extend the work of [7] by considering the channel estimation and prediction problem as a 2-dimensional frequency estimation problem. This approach is attractive in OFDM systems where pilot subcarriers in both the time and frequency domains are used. By using a two-dimensional parameterization, we are able to assess the prediction performance for a particular subcarrier and prediction length on 4 parameters - the number of pilots in the time/frequency domains $\left(N_{t}\right.$ and $N_{f}$ ), and their corresponding pilot spacings ( $D_{t}$ and $D_{f}$ ). Also in contrast to [7], we use the invariance property of the maximum likelihood estimator (MLE) ${ }^{1}$ to derive a simple expression for the CRB on the prediction error, without explicitly resorting to first-order Taylor approximations to the MSE. Interestingly, the resulting expression using either method is identical, thus confirming the result of the previous work in a more rigorous manner.

Although useful in its own right, conventional CRB analysis requires Monte-Carlo generation of channel realizations to obtain results independent of a particular channel. And with the objective of investigating the effect of various channel prediction parameter configurations, this requires performing a Monte-carlo simulation for each parameter configuration. This is a heavy computational burden even for the case of offline analysis, especially when $N_{t}$ and $N_{f}$ are large. This motivated us to derive a simple closed-form expression for the lower bound on prediction MSE in the asymptotic case of large $N_{t}$ and $N_{f}$. This expression relates the lowest-achievable MSE for an unbiased estimator with the predictor design parameters in a simple manner, which is independent of any particular channel realization. This provides important insight into the design of OFDM channel predictors, without the need for expensive Monte-Carlo numerical calculations. It is

\footnotetext{
${ }^{1}$ The invariance property for ML allows us to construct the MLE for a function of parameters $\boldsymbol{\theta}$, i.e. $\mathbf{f}=F(\boldsymbol{\theta})$, by computing the MLE of the parameters and using it in the function, i.e. $\hat{\mathbf{f}}=F(\hat{\boldsymbol{\theta}})[8, \mathrm{Ch} .6 .3]$.
} 
also shown through numerical examples that the asymptotic expression is reasonably tight for typical channel parameters with moderate values for $N_{t}$ and $N_{f}$, further motivating the use of these simple expressions.

\section{System Model}

\section{A. Time- and Frequency-Selective Wireless Channel}

The continuous-time impulse response of a time- and frequency-selective wireless channel can be modeled as the superposition of a discrete number of resolvable paths [9]

$$
h_{c}(t, \eta)=\sum_{p=0}^{L-1} \gamma_{p}(t) \delta\left(\eta-\eta_{p}(t)\right)
$$

where $\eta_{p}(t)$ and $\gamma_{p}(t)$ are the time-varying delay and complex gain corresponding to the $p$ th path, and $L$ is the number of propagation paths.

We assume that the wireless propagation environment has reflectors and scatterers that are far enough from the receiver, such that the waves incident on the receiving antenna are plane waves having a common angle of incidence. We also assume that the receiver travels in a linear motion and with constant velocity, such that the Doppler frequency is time independent. ${ }^{2}$ These assumptions allow us to express $\gamma_{p}(t)$ to be fitted by a linear combination of complex sinusoidal rays

$$
\gamma_{p}(t)=\sum_{r=0}^{M_{p}-1} \alpha_{r, p} e^{j \nu_{r, p}} e^{j 2 \pi \xi_{r, p} t}
$$

where $M_{p}$ is the number of rays contributing to the $p$ th path, $\alpha_{r, p}$ is the amplitude, $\nu_{r, p}$ is the phase, and $\xi_{r, p}$ is the Doppler frequency, respectively, for the $r$ th ray in the $p$ th path. A similar model has been used by various researchers for wireless channel characterization and prediction [7] [10] [11].

Substituting (2) into (1), combining indices, and taking its Fourier transform, we get the frequency response of the timevarying channel as

$$
H_{c}(t, f)=\sum_{i=1}^{M} \alpha_{i} e^{j \nu_{i}} e^{j 2 \pi\left(\xi_{i} t-\eta_{i} f\right)}
$$

where $M=\sum_{p=0}^{L-1} M_{p}$ is the total number of complex sinusoidal rays, and each ray is characterized by the quadruplet $\left\{\alpha_{i}, \nu_{i}, \xi_{i}, \eta_{i}\right\}$. We assume that no two rays share the same pair of time-delay and Doppler frequency values $\left\{\xi_{i}, \eta_{i}\right\}$ (otherwise they can be combined to form one ray), but different rays may share the same delay or Doppler frequency. Since practical values of $\xi_{i}$ and $\eta_{i}$ are bounded, i.e. $0 \leq \xi_{i}<\xi_{\max }$ and $0 \leq \eta_{i}<\eta_{\max }$, (3) has finite support in both Doppler and delay domains. Assuming that the OFDM system with symbol period $T_{\text {sym }}$ and subcarrier spacing $\Delta f$ has proper cyclic extension and sample timing, the sampled channel frequency

\footnotetext{
${ }^{2}$ These assumptions are valid in outdoor propagation scenarios, e.g mobile cellular environments; and in considering small time windows of interest, i.e. a few wavelengths.
}

response at the $k$ th tone of the $n$th OFDM block can be expressed as a linear combination of 2-dimensional complex sinusoidal rays

$$
\begin{aligned}
H(n, k) & \triangleq H_{c}\left(n T_{\text {sym }}, k \Delta f\right) \\
& =\sum_{i=0}^{M-1} \alpha_{i} e^{j \nu_{i}} e^{j 2 \pi\left(f_{i} n-\tau_{i} k\right)}
\end{aligned}
$$

where $f_{i}=\xi_{i} T_{\text {sym }}$ is the normalized Doppler frequency in radians, and $\tau_{i}=\eta_{i} \Delta f$ is the normalized frequency shift due to the time delay.

\section{B. OFDM Baseband Model}

We assume an OFDM system where the cyclic-prefix time $T_{c p}$ is greater than the maximum delay spread of the channel $\tau_{\max }$, the timing and carrier synchronization is perfect, and the used subcarriers are within the flat region of the frequency response of both transmit and receiver pulse-shaping filters, such that the received signal at the $k$ th subcarrier of the $n$th symbol can be written as

$$
\begin{aligned}
Y(n, k)= & H(n, k) X(n, k)+W(n, k) \\
& -N_{\text {used }} / 2 \leq k \leq N_{\text {used }} / 2, \quad k \neq 0
\end{aligned}
$$

where $W(n, k)$ is a circular-symmetric complex additive white Gaussian noise (AWGN) with variance $\sigma^{2}$.

Furthermore, we assume that there are $N_{f}$ equally spaced pilot subcarriers in every OFDM symbol inserted within the $N_{\text {used }}$ used subcarriers. Let $D_{f}=\left\lfloor N_{\text {used }} / N_{f}\right\rfloor$ denote the frequency spacing in terms of the number of subcarriers between two adjacent pilot subcarriers, where

$$
D_{f} \leq 1 /\left(\Delta f \tau_{\max }\right)
$$

to avoid aliasing in the frequency domain. We also let $\mathcal{K} \triangleq$ $\left\{k_{0}, \ldots, k_{N_{f}-1}\right\}$ denote the set of pilot subcarrier indices, where

$$
k_{q}=\left(\frac{N_{f}}{2}-q\right) D_{f}, \quad q=0, \ldots, N_{f}-1
$$

We also assume that a block of $N_{t}$ current and previous OFDM symbols (which we call pilot symbols) equally spaced in time are available for the channel prediction algorithm. Let $D_{t}$ denote the time spacing in terms of the number of OFDM symbols between pilot symbols, and where

$$
D_{t} \leq 1 /\left(2 T_{\text {sym }} f_{\max }\right)
$$

in order to avoid aliasing in the time domain. We also let $\mathcal{N} \triangleq\left\{n_{0}, \ldots, n_{N_{t}-1}\right\}$ denote the set of pilot symbol indices, where

$$
n_{l}=l D_{t}, \quad l=0, \ldots, N_{t}-1
$$

Using these $N_{t} \times N_{f}$ pilot subcarriers, we can perform a least-squares estimate of the channel at the pilot locations using the received signal $Y\left(n_{l}, k_{q}\right)$ and the known pilot symbols $X\left(n_{l}, k_{q}\right)$, given as

$$
\begin{aligned}
& \hat{H}_{L S}(l, q)=\sum_{i=0}^{M-1} \alpha_{i} e^{j \phi_{i}} e^{j\left(\omega_{i} l+\varphi_{i} q\right)}+\widetilde{W}(l, q), \\
& l=0, \ldots, N_{t}-1 \quad q=0, \ldots, N_{f}-1
\end{aligned}
$$


where

$$
\begin{aligned}
\phi_{i} & =\nu_{i}-2 \pi \tau_{i} D_{f} \frac{N_{f}-1}{2} \\
\omega_{i} & =2 \pi f_{i} D_{t} \\
\varphi_{i} & =2 \pi \tau_{i} D_{f}
\end{aligned}
$$

The basic premise in OFDM channel prediction is to simply use the least squares estimates of the channel (10) to in turn estimate the parameters in the channel model, i.e. $\alpha_{i}, \phi_{i}, \omega_{i}$, and $\varphi_{i}$. Channel prediction is then performed through simply extrapolating this model. Note that this idea has been used by several researchers [12] [11], albeit for narrowband, flat-fading channels. In the following section, we derive bounds on the MSE using this approach to OFDM channel prediction.

\section{Prediction MeAn-SQuare ERror Bounds}

Observe that (10) is in the standard form of a twodimensional complex sum-of-sinusoids in additive white Gaussian noise. Estimating the parameters of this model is a classical problem, and is very well studied in the radar, sonar imaging, and other array signal processing literature [13]. Hence, we can use classical results on the CRB for 2-D sinusoidal estimation to derive the CRB for OFDM channel prediction.

\section{A. OFDM Channel Prediction CRB}

Let

$$
\boldsymbol{\theta}=\left[\boldsymbol{\theta}_{0}^{T}, \ldots, \boldsymbol{\theta}_{M-1}^{T}\right]^{T}
$$

with

$$
\boldsymbol{\theta}_{i}=\left[\alpha_{i}, \phi_{i}, \omega_{i}, \varphi_{i}\right]^{T}
$$

denote the $4 M$-length vector of channel parameters which we estimate from (10).

Let us rewrite the physical channel model (4) in terms of the parameters we estimate, i.e.

$$
H(n, k, \boldsymbol{\theta})=\sum_{i=0}^{M-1} H_{i}\left(n, k, \boldsymbol{\theta}_{i}\right)
$$

with

$$
H_{i}\left(n, k, \boldsymbol{\theta}_{i}\right)=\alpha_{i} e^{j\left(\phi_{i}+\varphi_{i} \frac{N_{f}-1}{2}\right)} e^{j\left(\frac{\omega_{i}}{D_{t}} n-\frac{\varphi_{i}}{D_{f}} k\right)}
$$

Note that the dependence of the channel response on the parameter vectors (11) is made explicit in our notation.

Since our channel model is a continuous function of the unknown parameters, by the invariance principle of the maximum likelihood estimate, we can bound the prediction meansquare error using the Cramér-Rao lower bound for function of parameters [8, Ch. 6.4], i.e.

$$
\begin{aligned}
e(n, k) & =\mathbb{E}\left\{|H(n, k, \boldsymbol{\theta})-H(n, k, \hat{\boldsymbol{\theta}})|^{2}\right\} \\
& \geq \frac{\partial H(n, k, \boldsymbol{\theta})}{\partial \boldsymbol{\theta}} \mathbf{I}_{2 D}^{-1}(\boldsymbol{\theta}) \frac{\partial H(n, k, \boldsymbol{\theta})}{\partial \boldsymbol{\theta}}
\end{aligned}
$$

where

$$
\frac{\partial H(n, k, \boldsymbol{\theta})}{\partial \boldsymbol{\theta}}=\left[\begin{array}{c}
\frac{\partial H_{1}\left(n, k, \boldsymbol{\theta}_{1}\right)}{\partial \boldsymbol{\theta}_{1}} \\
\vdots \\
\frac{\partial H_{M-1}\left(n, k, \boldsymbol{\theta}_{M-1}\right)}{\partial \boldsymbol{\theta}_{M-1}}
\end{array}\right]
$$

with

$$
\frac{\partial H_{i}\left(n, k, \boldsymbol{\theta}_{i}\right)}{\partial \boldsymbol{\theta}_{i}}=H_{i}\left(n, k, \boldsymbol{\theta}_{i}\right)\left[\begin{array}{c}
\frac{1}{\alpha_{i}} \\
j \\
j \frac{n}{D_{t}} \\
-j \frac{k-\bar{k}}{D_{f}}
\end{array}\right]
$$

where $\bar{k}=\left(\frac{N_{f}-1}{2}\right) D_{f}$ and $\mathbf{I}_{2 D}^{-1}(\boldsymbol{\theta})$ is the well-known Cramér-Rao bound matrix for 2-dimensional sinusoidal parameter estimation, which can be found in [14, Appendix A]. An interesting point to note is that [7] and [11] came up with a similar MSE bound as (15), using a different and more tedious approach (first-order Taylor expansion for the MSE function). Thus, using the invariance principle, we were able to verify their result using a simpler approach.

\section{B. Asymptotic $C R B$}

Although the bound (15) can be used to study the effects of various parameters on the prediction MSE, its expression is not readily interpretable. Furthermore, since the bound depends on the actual parameter vector $\boldsymbol{\theta}$, Monte-Carlo simulations that generate realizations of this parameter vector assuming a certain probability distribution is required to assess the performance. And with the objective of investigating the effect of various channel prediction parameter configurations, this requires performing a Monte-carlo simulation for each parameter configuration. Note that approximately $8 M^{2} N_{t} N_{f}+$ $O\left((4 M)^{3}\right)$ operations are required to compute the CRB for each channel realization. This is a heavy computational burden even for the case of offline analysis, specially when $M, N_{t}$, and $N_{f}$ are large. This motivated us to derive a simple closedform expression for the lower bound on prediction MSE in the asymptotic case of large $N_{t}$ and $N_{f}$.

Thus, we consider the asymptotic MSE given as

$$
\begin{aligned}
\tilde{\epsilon}(n, k) & \geq \lim _{\min \left(N_{t}, N_{f}\right) \rightarrow \infty} \epsilon(n, k) \\
& =\frac{\partial H(n, k, \boldsymbol{\theta})}{\partial \boldsymbol{\theta}} \tilde{\mathbf{I}}_{2 D}^{-1} \frac{\partial H(n, k, \boldsymbol{\theta})}{\partial \boldsymbol{\theta}}
\end{aligned}
$$

where

$$
\tilde{\mathbf{I}}_{2 D}^{-1}=\left[\begin{array}{cccc}
\mathbf{K}_{1} & \mathbf{0} & \cdots & \mathbf{0} \\
\mathbf{0} & \mathbf{K}_{2} & \cdots & \mathbf{0} \\
\vdots & \vdots & \ddots & \vdots \\
\mathbf{0} & \mathbf{0} & \cdots & \mathbf{K}_{M}
\end{array}\right]
$$

is the block diagonal asymptotic CRB for 2-D superimposed exponential signals, with diagonal elements given by [15]

$$
\mathbf{K}_{i}=\left[\begin{array}{cccc}
\frac{\sigma^{2}}{2 N_{t} N_{f}} & 0 & 0 & 0 \\
0 & \frac{7 \sigma^{2}}{2 \alpha_{i}^{2} N_{t} N_{f}} & \frac{-3 \sigma^{2}}{\alpha_{i}^{2} N_{t}^{2} N_{f}} & \frac{-3 \sigma^{2}}{\alpha_{i}^{2} N_{t} N_{f}^{2}} \\
0 & \frac{-3 \sigma^{2}}{\alpha_{i}^{2} N_{t}^{2} N_{f}} & \frac{6 \sigma^{2}}{\alpha_{i}^{2} N_{t}^{3} N_{f}} & 0 \\
0 & \frac{-3 \sigma^{2}}{\alpha_{i}^{2} N_{t} N_{f}^{2}} & 0 & \frac{6 \sigma^{2}}{\alpha_{i}^{2} N_{t} N_{f}^{3}}
\end{array}\right]
$$


After further simplification, we have

$$
\begin{aligned}
\tilde{\epsilon}(n, k) \geq \sigma^{2} M( & \frac{4}{N_{t} N_{f}}-\frac{6 n}{N_{t}^{2} N_{f} D_{t}}+\frac{6 n^{2}}{N_{t}^{3} N_{f} D_{t}^{2}} \\
& \left.+\frac{6(k-\bar{k})}{N_{t} N_{f}^{2} D_{f}}+\frac{6(k-\bar{k})^{2}}{N_{t} N_{f}^{3} D_{f}^{2}}\right)
\end{aligned}
$$

Note that this bound is tightest when $N_{t}$ and $N_{f}$ are large, or equivalently the spacing of the frequencies are much larger than the resolution limit, i.e. $\left|\tau_{i}-\tau_{j}\right| \gg 1 / N_{f}$ and $\left|f_{i}-f_{j}\right| \gg$ $1 / N_{t}$. Note also that this bound is actually achievable by the maximum likelihood method [15] and nonlinear-least squares method [16] in the large sample limit.

Using this simple expression for the lower bound on MSE, we could easily deduce the impact of the various parameters on the MSE, e.g.

- The bound increases linearly with increasing noise variance $\sigma^{2}$ and number of 2-D sinusoidal rays $M$. This is intuitively satisfying, and agrees with previous results that dense multipath channel environments, i.e. $M$ large, are the hardest to predict [11].

- The contribution to the overall MSE from the error variances corresponding to the estimate of frequencies $\omega_{i}$ and $\varphi_{i}$ grow quadratically with $n$ and $|k|$, emphasizing the importance of estimating these accurately.

- In general, $N_{t}$ and $N_{f}$ and the downsampling factors $D_{t}$ and $D_{f}$ should be chosen as large as possible to decrease the MSE bound, but are of course subject to limitations imposed by other system consideration like complexity, training overhead, validity of the linear model, etc.

\section{NUMERICAL RESUltS}

The purpose of this section is to study the effect of the various parameters on the OFDM prediction MSE bounds using numerical evaluation on a computer. In order to be as concrete and as realistic as possible, we provide numerical results for an outdoor mobile OFDM system based on the IEEE 802.16e mobile broadband wireless access standard [17], whose parameters are given in Table I.

We consider a wireless channel based on the ITU Vehicular A [18] power delay profile, which is a sparse multipath model with 6 taps, each tap having Clarke's classical U-shaped spectrum [9]. We assume that each path is composed of 16 complex sinusoidal rays, whose amplitude, phase, and Doppler frequency parameters are generated using the modified Jake's simulation model [19].

TABLE I

Simulation PARAMETERS

\begin{tabular}{c|c}
\hline Parameter & Value \\
\hline \hline Bandwidth $(\mathrm{B})$ & $5 \mathrm{MHz}$ \\
\hline Sampling Frequency $\left(f_{s}\right)$ & $5.712 \mathrm{Ms} / \mathrm{s}$ \\
\hline Carrier Frequency $\left(f_{c}\right)$ & $2.4 \mathrm{GHz}$ \\
\hline Mobile Velocity $(v)$ & $75 \mathrm{~km} / \mathrm{hr}$ \\
\hline Maximum Doppler Frequency $\left(f_{\max }\right)$ & $166.67 \mathrm{~Hz}$ \\
\hline$N_{f f t}$ & 512 \\
\hline$N_{\text {used }}$ & 426 \\
\hline
\end{tabular}

Using the above channel parameters, the downsampling factors should be chosen as $D_{f} \leq 35$ (6) and $D_{t} \leq 29$ (8) to avoid aliasing in both domains. We also require the pilot subcarriers in the frequency domain to be within the used subcarriers $N_{f} D_{f} \leq N_{\text {used }}=426$. The restriction on $N_{t} D_{t}$ however, requires more knowledge on the exact scattering environment, since this value should be confined to the interval over which the linear approximation (2) is valid. In [20], a rough approximation to this interval based on the accumulated phase error in the sinusoidal model is given as

$$
T=\sqrt{\frac{\lambda r}{3 v^{2}}}
$$

where $\lambda$ is the wavelength, $r$ is the distance of the mobile to the closest scatterer, and $v$ is the mobile velocity. We estimate the distance of the scatter as $r=\tau_{r m s} c$ where $\tau_{r m s}$ is the root-mean-square delay spread [9], and $c=3 \times 10^{8} \mathrm{~m} / \mathrm{s}$ is the speed of light. Using these approximations, the interval over which the channel model is valid is approximately $17 \lambda$ or 1024 OFDM symbols. Thus, we require $N_{t} D_{t}+n \leq 1024$, where $n$ is the prediction length.

Figure 1 shows the MSE bounds for subcarrier $k=N_{\text {used }} / 2$ and prediction length $n=D_{t}$ versus the number of pilots used in time $\left(N_{t}\right)$ and frequency $\left(N_{f}\right)$ domains. The upper surface is the CRB, and the lower surface is the Asymptotic CRB. We set $N_{t} D_{t}=1024$ and $N_{f} D_{f}=426$ to satisfy parameter restrictions, relaxing the integer requirement on $D_{t}$ and $D_{f}$. The MSE clearly decreases as we increase both $N_{t}$ and $N_{f}$. For values of $N_{t}<40$ and for values of $N_{f}<10$, the surface is not plotted since the Fisher information matrix in this case is singular. This is because we have $4 M=4 \times 6 \times 16=384$ parameters to estimate, requiring $N_{t} N_{f}>384$ in order for the problem to be statistically well-posed. Furthermore, note that for reasonable values of $N_{t}$ and $N_{f}$, the asymptotic CramerRao bound is close to the CRB, and the two bounds become closer as we increase $N_{t}$ and $N_{f}$ as expected.

Figure 2 shows the MSE bounds for all the used subcarriers versus the $D_{t}$, where the prediction length $n=60(\approx 1 \lambda)$, and $N_{t}=50, N_{f}=42, D_{f}=10$. Once again, the upper surface is the CRB, and the lower surface is the asymptotic CRB. Note that increasing $D_{t}$ while holding $N_{t}$ fixed decreases the MSE as expected. This is an important concept since increasing $D_{t}$ does not cost extra in terms of computations, and is thus a good tool to improve MSE performance without increasing complexity. The asymptotic CRB is also tight in this case.

Figure 3 shows the MSE bounds for all subcarriers versus prediction length expressed in wavelengths, where the upper and lower surfaces correspond to the CRB and asymptotic CRB respectively. We set $N_{t}=100, D_{t}=19, N_{f}=141$, and $D_{f}=3$. These parameters coincide with the $2 \mathrm{~ms}$ frame size for IEEE 802.16e, and assuming that we perform an estimate in every preamble of the frame which has 141 pilots spaced 3 subcarriers apart. Note the quadratic nature of the MSE versus subcarrier index, showing that the edge subcarriers have the highest prediction MSE. This can be thought of as an alternative explanation for the so called edge effect in OFDM 


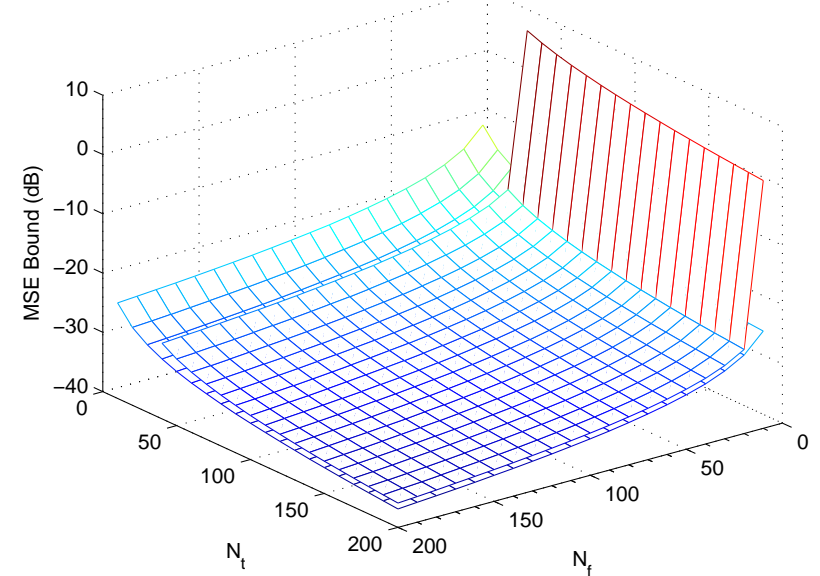

Fig. 1. MSE bounds for subcarrier $k=N_{\text {used }} / 2$ and prediction length $n=D_{t}$ versus the number of pilots used in time $\left(N_{t}\right)$ and frequency $\left(N_{f}\right)$ domains. The upper surface is the Cramer-Rao bound, and the lower surface is the asymptotic CRB. We set $N_{t} D_{t}=1024$ and $N_{f} D_{f}=426$ to satisfy parameter restrictions, relaxing the integer requirement on $D_{t}$ and $D_{f}$.

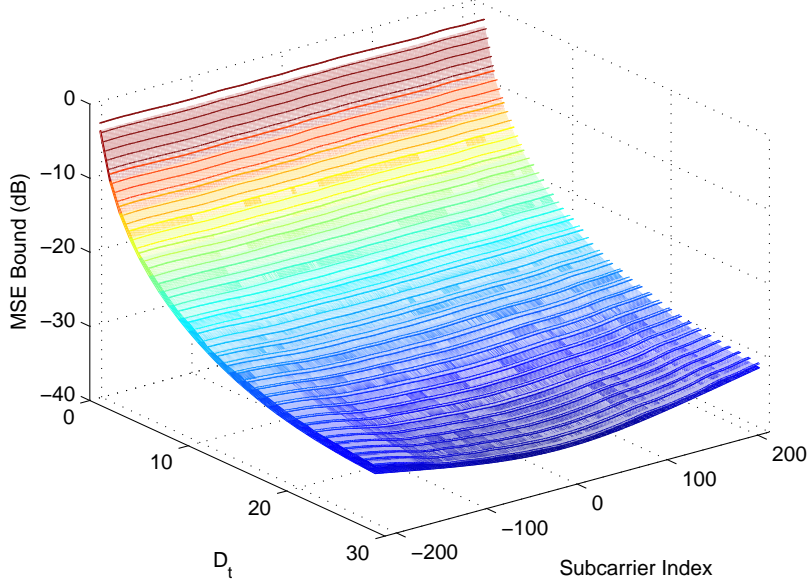

Fig. 2. MSE bounds for all subcarriers for various $D_{t}$, for a prediction length $n=60(\approx 1 \lambda)$ and $N_{t}=100, N_{f}=42, D_{f}=10$. The upper surface is the CRB, and the lower surface is the Asymptotic CRB.

channel estimation. We also note the quadratic increase of the MSE bound with prediction length.

\section{REFERENCES}

[1] T. Keller and L. Hanzo, "Adaptive modulation techniques for duplex OFDM transmission," IEEE Trans. Veh. Technol., vol. 49, no. 5, pp. 1893-1906, 2000

[2] M. R. Souryal and R. L. Pickholtz, "Adaptive Modulation with Imperfect Channel Information in OFDM," in Proc. IEEE Int. Conf. Comm., 2001, pp. 1861-1865.

[3] I. C. Wong and B. L. Evans, "Joint Channel Estimation and Prediction for OFDM Systems," in Proc. IEEE Global Telecommunications Conference, St. Louis, MO, 2005.

[4] A. Forenza and R. W. Jr. Heath, "Link Adaptation and Channel Prediction in Wireless OFDM Systems," in Proc. IEEE Midwest Symp. on Circuits and Sys., 2002, pp. 211-214.

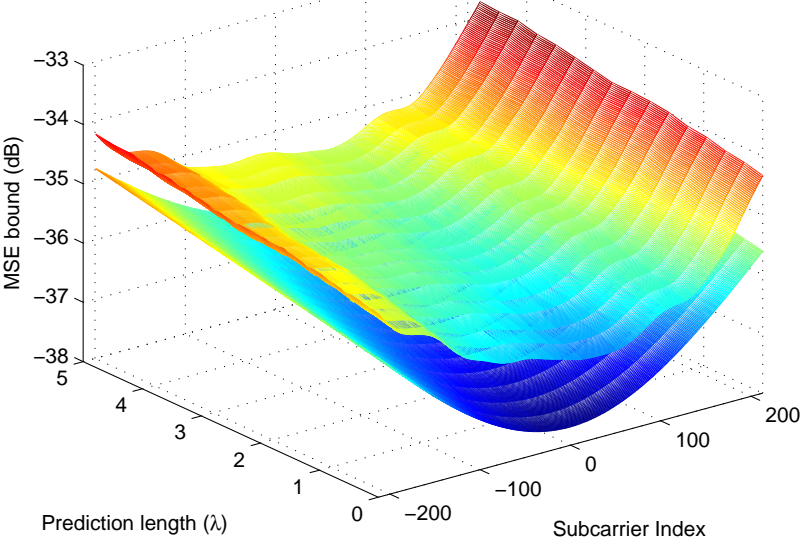

Fig. 3. MSE bounds for all subcarriers versus prediction length expressed in wavelengths, for $N_{t}=100, D_{t}=19, N_{f}=141, D_{f}=3$. The upper surface is the CRB, and the lower surface is the Asymptotic CRB.

[5] M. Sternad and D. Aronsson, "Channel estimation and prediction for adaptive OFDM downlinks [vehicular applications]," in Proc. IEEE Vehicular Technology Conference, vol. 2, 2003, pp. 1283-1287.

[6] D. Schafhuber and G. Matz, "MMSE and Adaptive Prediction of TimeVarying Channels for OFDM Systems," IEEE Trans. Wireless Commun., vol. 4, no. 2, pp. 593-602, Mar 2005.

[7] S. Barbarossa and A. Scaglione, "Theoretical bounds on the estimation and prediction of multipath time-varying channels," in Proc. IEEE International Conference on Acoustics, Speech, and Signal Processing, vol. 5, 2000, pp. 2545-2548.

[8] L. L. Scharf and C. Demeure, Statistical signal processing : detection, estimation, and time series analysis. Addison-Wesley Pub. Co., 1991.

[9] T. S. Rappaport, Wireless communications : principles and practice, 2nd ed. Prentice Hall, 2002.

[10] A. Duel-Hallen, S. Hu, and H. Hallen, "Long-Range Prediction of Fading Signals," IEEE Signal Processing Mag., vol. 17, no. 3, pp. 62-75, May 2000.

[11] P. D. Teal, "Real time characterisation of the mobile multipath channel," Ph.D. dissertation, Australian National University, 2002.

[12] J. K. Hwang and J. H. Winters, "Sinusoidal modeling and prediction of fast fading processes," in Proc. IEEE Global Telecommunications Conference, 1998, pp. 892-897.

[13] S. M. Kay, Modern Spectral Estimation : Theory and Application. Prentice Hall, 1988.

[14] Y. Hua, "Estimating two-dimensional frequencies by matrix enhancement and matrix pencil," IEEE Trans. Signal Processing, vol. 40, no. 9, pp. 2267-2280, Sep 1992.

[15] C. Rao, L. Zhao, and B. Zhou, "Maximum likelihood estimation of 2D superimposed exponential signals," IEEE Trans. Signal Processing, vol. 42, no. 7, pp. 1795-1802, Jul 1994.

[16] A. Mitra and P. Stoica, "The Asymptotic Cramer-Rao Bound for 2D Superimposed Exponential Signals," Multidimensional Systems and Signal Processing, vol. 13, no. 3, pp. 317-331, Jul 2002.

[17] Air Interface for Fixed and Mobile Broadband Wireless Access Systems, IEEE Std. 802.16e/D5, 2004.

[18] Selection procedures for the choice of radio transmission technologies for the UMTS, ETSI Std. TR 101112 v. 3.2.0, 1998.

[19] Y. R. Zheng and C. Xiao, "Simulation models with correct statistical properties for Rayleigh fading channels," IEEE Trans. Commun., vol. 51, no. 6 , pp. 920-928, Jun 2003.

[20] T. Ekman, "Prediction of mobile radio channels," Ph.D. dissertation, Uppsala University, 2002. 